\title{
The intervals of oscillations in the solutions of the radial Schrödinger differential equation
}

\author{
Qutaibeh D Katatbeh ${ }^{1 *}$, Dimitris M Christodoulou² and James Graham-Eagle²
}

\section{"Correspondence:}

qutaibeh@yahoo.com

'Department of Mathematics and

Statistics, Jordan University of

Science and Technology, Irbid, 22110, Jordan

Full list of author information is available at the end of the article

\begin{abstract}
We have previously formulated a program for deducing the intervals of oscillations in the solutions of ordinary second-order linear homogeneous differential equations. In this work, we demonstrate how the oscillation-detection program can be carried out for the radial Schrödinger equation when a Coulomb potential is used to describe the hydrogen atom. The method predicts that the oscillation intervals are finite in radius and their sizes are determined uniquely by the two quantum numbers $n$ and $\ell$. Numerical integrations using physical boundary conditions at the origin confirm this oscillatory behavior of the radial Coulomb wavefunctions. Two related differential equations due to Kummer and Whittaker and other attractive electrostatic potentials are also discussed in the same context.
\end{abstract}

MSC: 34A25; 34A30; 81Q05

Keywords: oscillations; second-order linear differential equations; analytical theory; transformations; Schrödinger differential equation; Kummer's differential equation; Whittaker differential equation

\section{Introduction}

All ordinary second-order linear homogeneous differential equations can be written in the general form

$$
y^{\prime \prime}+b(x) y^{\prime}+c(x) y=0
$$

and this equation can be transformed to the canonical form

$$
u^{\prime \prime}+q(x) u=0
$$

where the primes denote derivatives with respect to the independent variable $x, q=$ $-\left(b^{2}+2 b^{\prime}-4 c\right) / 4$, and $y(x)=u(x) \exp \left(-\frac{1}{2} \int b(x) d x\right)$ (see, e.g., Section 10.2 in [1] and Section XI.1 in [2]). The canonical form (2) condenses the coefficients of equation (1) into a single coefficient $q(x)$ and 'oscillation theory' focuses mainly on $q(x)$ in order to deduce the oscillatory properties of the solutions of equation (1) (see the reviews in $[3,4]$ and references therein). In a previous paper ([5], hereafter CGK), we also studied the $q(x)$ of equation (2) in order to find out what information it may reveal about the solutions of

(c) 2016 Katatbeh et al. This article is distributed under the terms of the Creative Commons Attribution 4.0 International License (http://creativecommons.org/licenses/by/4.0/), which permits unrestricted use, distribution, and reproduction in any medium, provided you give appropriate credit to the original author(s) and the source, provide a link to the Creative Commons license, and indicate if changes were made. 
equation (1). First, we formulated a new definition of oscillation as the appearance of successive critical points of the same kind (maxima, or minima, or inflection points) in the graph of a solution. Then we described a program for deducing the precise intervals of oscillations in the solutions of the general form (1), and we presented a variety of examples in which this procedure was successful in predicting oscillatory behavior solely from an examination of the coefficient $q(x)$ of equation (2).

Some of the differential equations that were investigated by CGK were transformed to the harmonic-oscillator form with constant coefficients in the first step of the program, and their oscillatory properties were then derived easily from the discriminant of their characteristic quadratic equations. The rest of the equations were transformed to a form with constant damping (a constant coefficient of the first-derivative term), a quantity that clearly opposes any oscillatory tendencies in the solutions. In this second step of the program, a generalized Euler transformation of the independent variable $x$ was used:

$$
x=c_{1}+c_{2} \exp (k t)
$$

where $c_{1}, c_{2}$, and $k$ are arbitrary constants, and a criterion for the intervals of oscillations in the solutions was established:

$$
q(x)>\frac{1}{4\left(x-c_{1}\right)^{2}} .
$$

Only the constant $c_{1}$ appears in the criterion and corresponds to a 'horizontal shift' of the independent variable $x$ in equation (3). For equations with singularities at the origin, $c_{1}$ can be set to zero, and then criterion (4) takes the form

$$
q(x)>\frac{1}{4 x^{2}}
$$

In this case, we can also choose $c_{2}=1$ and $k=1$ in equation (3), and then the change of the independent variable $x$ takes the form of the classical Euler transformation

$$
x=\exp (t)
$$

for which the investigation of the interval $t \in(-\infty,+\infty)$ in the transformed equation corresponds to searching for oscillatory solutions in the interval $x \in(0,+\infty)$ of the original equation (1).

In the present work, we extend our investigation to the study of oscillatory properties of the radial Schrödinger equation that is of interest to the field of quantum mechanics (see, e.g., Chapter 14 in [6] and Section 67 in [7]). In Section 2, we adopt a classical Coulomb potential of the form $V(x) \propto-1 / x$ that describes the hydrogen atom (as in Section 67 of [7]), and we transform the radial Schrödinger equation into the canonical form (2). Then we use the formulation expressed by equations (5) and (6) to analyze the properties of the resulting coefficient $q(x)$ in the interval $x \in(0,+\infty)$. The same $q(x)$ appears also in the canonical forms of Kummer's equation [6,7] and Whittaker's equation [1, 6]. These closely related equations are discussed in Section 2.2. In Section 3, we carry out numerical integrations of the original equation (1) under physical boundary conditions at $x=0$ in 
order to test the predictions of the analytical formulation. Finally, in Section 4, we summarize our results and discuss their relevance to various two-particle quantum mechanical models.

\section{Radial Schrödinger equation with Coulomb potential}

The radial Schrödinger equation $[6,7]$ can be written in normalized units as

$$
y^{\prime \prime}+\frac{2}{x} y^{\prime}-\left[n V(x)+\frac{\ell(\ell+1)}{x^{2}}+\frac{1}{4}\right] y=0
$$

where the integers $n \geq 1$ and $0 \leq \ell \leq n-1$ are the principal and secondary quantum numbers, respectively, $V(x)$ is the normalized potential, and $x \geq 0$ is a scaled radial coordinate of the radial wavefunction $y(x)$. The canonical form (2) of equation (7) has $u(x)=x y(x)$, and its $q(x)$ is identical to the coefficient of $y$ because the coefficient of $y^{\prime}$ is precisely equal to $2 / x$ (this property was discussed in detail by CGK in their Section 6.2 ):

$$
q(x)=-\left[n V(x)+\frac{\ell(\ell+1)}{x^{2}}+\frac{1}{4}\right] .
$$

\subsection{The hydrogen atom}

For the normalized potential $V(x)$, we use the classical spherically symmetric Coulomb form:

$$
V(x)=-\frac{1}{x}
$$

in which case equation (8) becomes

$$
q(x)=\frac{n}{x}-\frac{\ell(\ell+1)}{x^{2}}-\frac{1}{4}
$$

Since $n$ is assumed to be a positive integer, only bound states of the electron in the hydrogen atom are examined, and their negative energy levels $E_{n}=-(13.6 \mathrm{eV}) / n^{2}$ are fully determined by the chosen values of $n$.

Equation (10) has a regular singular point at $x=0$, in which case the criterion for oscillatory solutions is described by inequality (5). Substituting now equation (10) into equation (5), we find that the interval over which the wavefunctions of equation (7) may oscillate is given by the solution of the algebraic inequality

$$
x^{2}-4 n x+(2 \ell+1)^{2}<0 \quad \Longrightarrow \quad x \in\left(x_{-}, x_{+}\right)
$$

where

$$
x_{ \pm}=2 n \pm \sqrt{4 n^{2}-(2 \ell+1)^{2}} .
$$

It is easy to show that $x_{ \pm}>0$ for $n \geq 1$ and $0 \leq \ell \leq n-1$. Therefore, oscillations appear only in a finite radial interval in each case in which $n$ and $\ell$ are chosen. There are however two limiting cases in which a complete oscillation (i.e., a full 'cycle') does not develop in such an interval: 
(a) We know from the results obtained by CGK that some low-order solutions of equation (7) are nonoscillatory and that the lowest-order solutions that may exhibit at least two critical points of the same kind (maxima, or minima, or inflection points) for certain choices of boundary conditions have $n=3$.

(b) For the two largest values of the secondary quantum number, $\ell=n-2$ and $\ell_{\max }=n-1$, the wavefunctions have only one node and no node, respectively, in the intervals determined by equation (11). Therefore, although the solutions are distinctly nonmonotonic in these intervals, they do not develop two critical points of the same kind, and they are then nonoscillatory according to the definition given in Section 1.

These limiting cases are discussed further in Section 3.

\subsection{Related equations and degeneracy of the canonical form}

It is important to note that the above criterion for oscillations is unique and robust. The exact same inequality as in equation (11) is also derived from alternative forms of the radial Schrödinger equation. We mention here two such characteristic cases. In the first case (Section 67 in [7]), the differential equation for the hydrogen atom is written in Kummer's form [6] of the confluent hypergeometric equation:

$$
y^{\prime \prime}+\left(\frac{2 \ell+2}{x}-1\right) y^{\prime}+\frac{n-\ell-1}{x} y=0 \quad(x>0)
$$

The structure of this equation is different than that of equation (7), but the coefficient $q(x)$ of its canonical form is the same as equation (10). Therefore, when condition (5) is applied to $q(x)$, the criterion for oscillations in the wavefunctions of equation (13) turns out to be the same as that derived for the wavefunctions of equation (7).

In the second case (Section 16.1 in [1]), we consider Whittaker's differential equation

$$
y^{\prime \prime}+y^{\prime}+\left(\frac{k}{x}+\frac{\frac{1}{4}-m^{2}}{x^{2}}\right) y=0 \quad(x>0),
$$

where $k$ and $m$ are constants. The canonical form (2) of this equation has a coefficient $q(x)$ that takes the form of equation (10) with $n=k$ and $\ell=m-1 / 2$. Therefore, when condition (5) is applied to $q(x)$, the criterion for oscillations in the negative-energy $(k>0 ;[8,9])$ solutions of Whittaker's equation (14) turns out to have the same form as that derived before for the wavefunctions of equation (7).

Equation (14) has a constant coefficient in its $y^{\prime}$ term, so a constant damping is folded into the canonical form (2). This is not the case for equations (7) and (13), whose transformations lead to the same canonical form. The fact that $q(x)$ takes the same form in these three cases implies that differential equations of the form (1) with markedly different structures in their coefficients can be cast to the same canonical form with virtually identical $q(x)$ coefficients by transformations of the dependent variable $y$. This is a kind of degeneracy in the canonical transformation of the general form (1). Because of this degeneracy, when we seek the oscillation intervals of a given differential equation in the form of equation (1) or equation (2), we are obliged to undertake the steps of the program described in CGK, irrespective of the symmetries that the given equation may exhibit (e.g., constant damping or being already in canonical form). In particular, the transformation of 
the independent variable $x$ described in Section 2.3 of CGK must be carried out before a criterion for oscillatory solutions can be established. This is because the transformation of $x$ is not degenerate: two canonical equations of the form (2) with different coefficients $q(x)$ do not produce the same final canonical form with coefficient $\hat{q}(x)=k^{2}\left[\left(x-c_{1}\right)^{2} q(x)-1 / 4\right]$, where $k$ and $c_{1}$ are arbitrary constants (see equations [16] and [17] in CGK).

\section{Numerical solutions}

Figures 1 and 2 show two numerical solutions of equation (7) for $n=5, \ell=2$ and $n=$ $10, \ell=7$, respectively, and with physical boundary conditions $y(0)=0$ and $y^{\prime}(0)=1$. In both cases, the solutions are oscillatory according to our definition since they exhibit two extrema of the same kind.

Figure 3 is analogous to Figure 2, except that $\ell=n-2=8$ is closer to the maximum value of $\ell_{\max }=9$. Although it is distinctly nonmonotonic in the interval specified by equation (12), this solution is nonoscillatory (two extrema of the same kind do not appear) precisely because $\ell=n-2$ (see below).

Additional numerical integrations of equation (7) confirm that (a) for values of $n \geq 3$ and $\ell \leq n-3$, the solutions exhibit increasingly more oscillations with increasing $n$ for a fixed value of $\ell$; and (b) for a fixed value of $n$, such oscillations become fewer with increasing $\ell$ and eventually disappear for $\ell=n-2$ and for $\ell_{\max }=n-1$.

The disappearance of oscillations for $n \leq 2$ and for $\ell \geq n-2$ can be understood in terms of the number of nodes $\mathcal{N}$ that the radial wavefunctions develop as a function of $n$ and $\ell$ :

$$
\mathcal{N}=n-\ell-1,
$$

This number is also called the 'radial quantum number' (Section 65 in [7]) and the boundary node at $x=0$ is not included. We distinguish the following four limiting cases:

(a) For $n=1$, then $\ell=0$, and the wavefunction is nodeless.

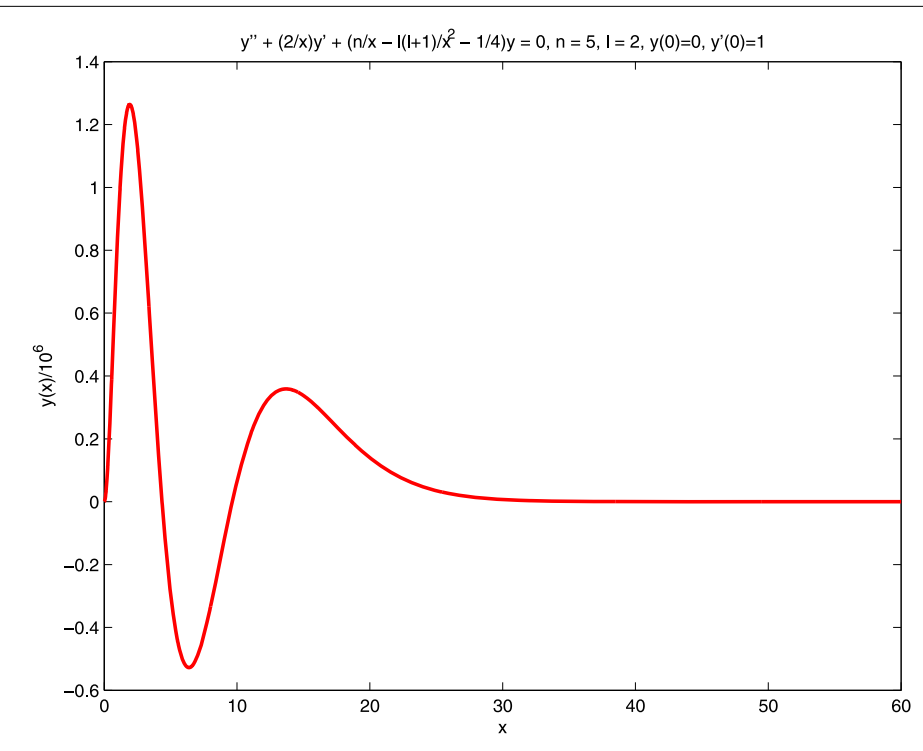

Figure 1 The numerical solution of equation (7) with $n=5, \ell=2$, and boundary conditions $y(0)=0$ and $y^{\prime}(\mathbf{0})=\mathbf{1}$. The solution oscillates in $x \in(1.34,18.66)$, as predicted by equation (12). 


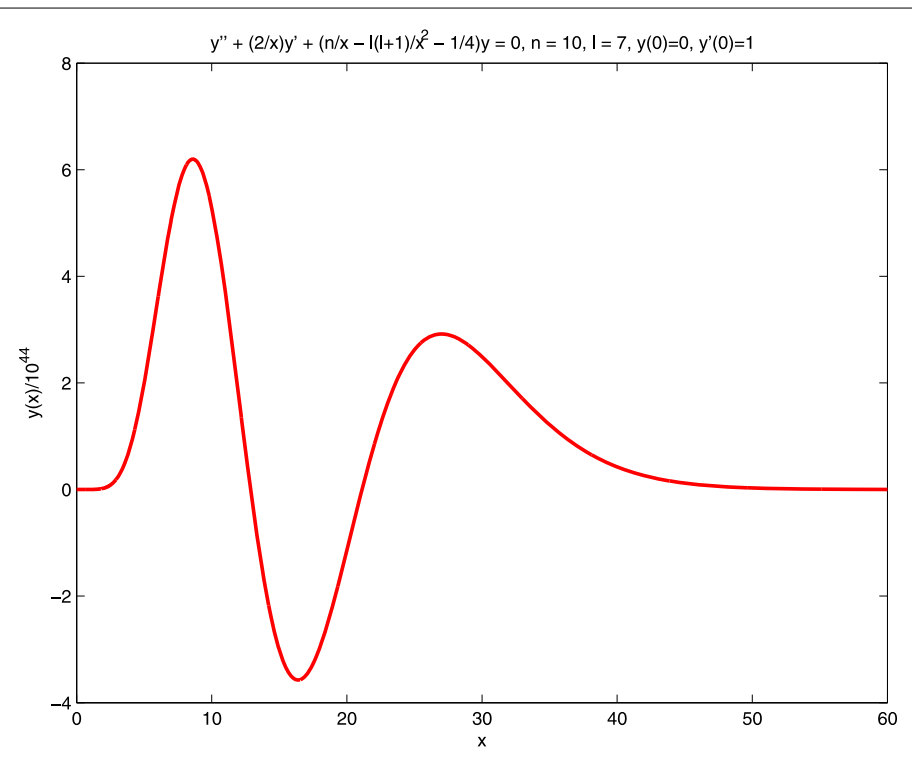

Figure 2 The numerical solution of equation (7) with $n=10, \ell=7$, and boundary conditions $y(0)=0$ and $y^{\prime}(0)=1$. The solution oscillates in $x \in(6.77,33.23)$, as predicted by equation (12).

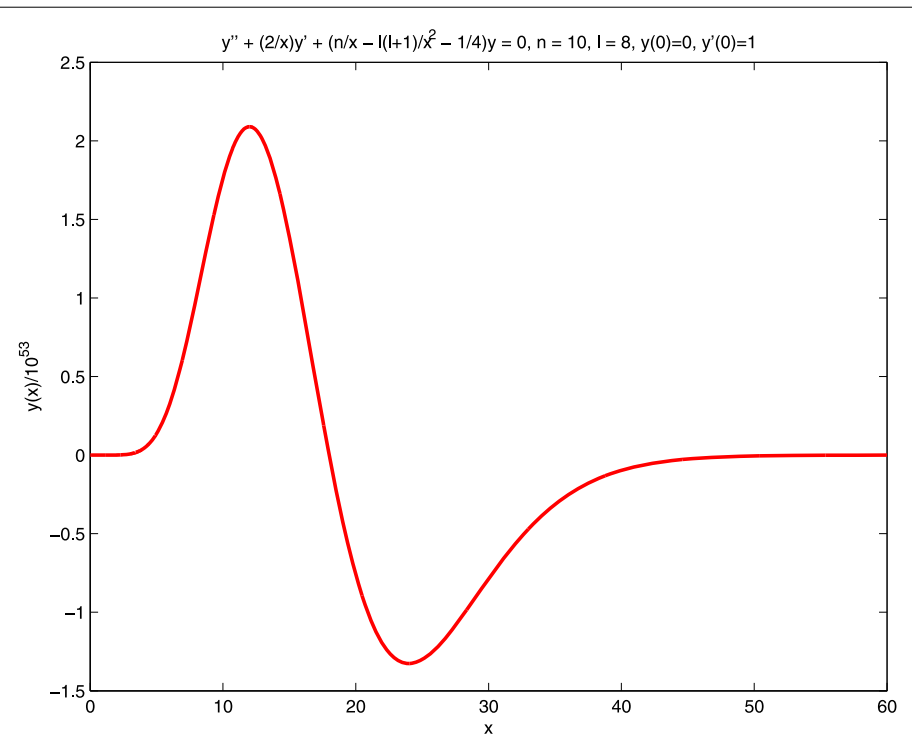

Figure 3 The numerical solution of equation (7) with $n=10, \ell=8$, and boundary conditions $y(0)=0$ and $y^{\prime}(\mathbf{0})=\mathbf{1}$. Despite its nonmonotonic behavior in the interval $x \in(9.46,30.54)$ specified by equation (12), this solution is nonoscillatory (two critical points of the same kind do not appear) because the value of $\ell$ is too close to the maximum value of $\ell_{\max }=9$.

(b) For $n=2$, then $\ell=0$ or 1 , and the wavefunctions have one node and no node, respectively.

(c) For $\ell=n-2$, then $\mathcal{N}=1$, and these wavefunctions have one node.

(d) For $\ell_{\max }=n-1$, then $\mathcal{N}=0$, and these wavefunctions are all nodeless.

In the cases with no node, the wavefunctions exhibit just one pronounced extremum, and they are clearly nonoscillatory. Furthermore, in the cases with $\mathcal{N}=1$, the wavefunctions exhibit a maximum and a minimum, but these extrema are not of the same kind; thus, 
these solutions are also nonoscillatory according to the definition given in Section 1. In all other cases with $\mathcal{N} \geq 2$, the wavefunctions exhibit two or more nodes, which, for the chosen boundary conditions, guarantee the appearance of two or more extrema of the same kind and thus oscillatory radial profiles.

\section{Summary and discussion}

\subsection{Summary}

In Section 2, we have presented an analysis of the oscillatory properties of the solutions of the radial Schrödinger equation (7) with a Coulomb potential of the form (9) that describes the hydrogen atom [6,7]. This equation has a regular singular point at $x=0$, and its canonical form has the same structure and properties as the canonical forms of the closely related Kummer [6, 7] and Whittaker [1, 6] equations (Section 2.2). Our analysis of these equations makes use of a program that was described in [5] by CGK for investigating the $x$-intervals in which the solutions exhibit an oscillatory character.

The main result of our investigation is that the radial Schrödinger wavefunctions of the classical Coulomb potential show oscillations only in a finite interval the extent of which is determined analytically and given by equation (12) in terms of the two quantum numbers $n$ and $\ell$. This result could not have been obtained by classical oscillation theory [2-4] because the Coulomb wavefunctions do not exhibit infinitely many nodes. Numerical integrations of equation (7) using physical boundary conditions $\left(y(0)=0\right.$ and $\left.y^{\prime}(0)=1\right)$ are described in Section 3, and they confirm the predicted oscillatory intervals in the wavefunctions for various choices of $n \geq 3$ and $\ell \leq n-3$ (two examples are shown in Figures 1 and 2).

On the other hand, there exist solutions in which one complete oscillation (a full 'cycle') does not develop in the intervals specified by equation (12). Even in such cases, however, the wavefunctions are distinctly nonmonotonic in the predicted intervals. Complete oscillations do not occur for low-order solutions $(n \leq 2)$ and for values of $\ell \geq n-2$ (a related example with $n=10$ and $\ell=8$ is shown in Figure 3). The wavefunctions in these cases exhibit at most one node in $x \in(0,+\infty)$, which is not enough for two extrema of the same kind to develop (see Section 3 for details).

\subsection{Discussion}

In quantum mechanics, the radial Schrödinger equation (7) is often studied for a variety of potentials other than the classical Coulomb potential. These include the Kratzer and Morse diatomic potentials [7], the two-particle Yukawa [7] and Hellmann [10] potentials, the cutoff Coulomb potential [11], and various power-law attractive potentials [12]. In all of these cases, the coefficient $q(x)$ of the canonical Schrödinger form is given by equation (8), where $V(x)$ is the particular type of the adopted potential, and the criterion for the appearance of oscillations in the wavefunctions is given by equation (5).

Combining equations (5) and (8), we find a general condition for oscillatory intervals in the solutions of the radial Schrödinger equation (7) for any of the potentials $V(x)$ previously mentioned:

$$
V(x)<V_{n \ell}^{*}(x) \equiv-\frac{1}{4 n}\left[1+\frac{(2 \ell+1)^{2}}{x^{2}}\right],
$$

where the integers $n \geq 1$ and $0 \leq \ell \leq n-1$ are the first two quantum numbers. Therefore, the adopted potential will exhibit oscillatory wavefunctions (or, at the very least, a distinct 
nonmonotonic behavior) only in the intervals in which it is more negative than the critical potential $V_{n \ell}^{*}(x)$. Specifically, in the ground state with $n=1$ and $\ell=0$, the critical potential takes the simple form

$$
V_{10}^{*}(x)=-\frac{1}{4}\left(1+\frac{1}{x^{2}}\right)
$$

but the $n=1$ wavefunction is nodeless and thus nonoscillatory (see Section 3 ). The lowestorder state that is expected to exhibit an oscillatory profile is the second excited state with $n=3$ and $\ell=0$ whose wavefunction develops two radial nodes (equation (15)). For this state, the critical potential is $\frac{1}{3} V_{10}^{*}(x)$, that is, less negative by a factor of 3 than the critical potential of the ground state.

On the other end of the spectrum, the wavefunctions of highly excited states with large values of $n$ and low-to-moderate values of $\ell$ will certainly exhibit oscillations since, for $n \gg 1$ and $\ell \ll \sqrt{n}$, the condition for oscillations (16) reduces asymptotically to $V(x)<0$, which is automatically satisfied for attractive electrostatic potentials. In the same case, however, the wavefunctions are expected to become nonoscillatory for $\ell \geq n-2$ because for the two largest values of $\ell$, the solutions of the radial Schrödinger equation (7) are not expected to have enough radial nodes (see equation (15) in Section 3).

Competing interests

The authors declare that they have no competing interests.

Authors' contributions

All authors drafted the manuscript, and they read and approved the submitted version.

\section{Author details}

'Department of Mathematics and Statistics, Jordan University of Science and Technology, Irbid, 22110, Jordan.

${ }^{2}$ Department of Mathematical Sciences, University of Massachusetts Lowell, Lowell, MA 01854, USA.

\section{Acknowledgements}

During this research project, DMC and JG-E were supported by the University of Massachusetts Lowell, whereas QDK was on a sabbatical visit and was fully supported by the Jordan University of Science and Technology.

Received: 25 October 2015 Accepted: 27 January 2016 Published online: 19 February 2016

\section{References}

1. Whittaker, ET, Watson, GN: A Course of Modern Analysis, 3rd edn. Cambridge University Press, Cambridge (1920)

2. Hartman, P: Ordinary Differential Equations. Wiley, New York (1964)

3. Agarwal, RP, Grace, SR, O'Regan, D: Oscillation Theory for Second Order Linear, Half-Linear, Superlinear and Sublinear Dynamic Equations. Kluwer Academic, Dordrecht (2002)

4. Wong, JSW: On second-order nonlinear oscillation. Funkc. Ekvacioj 11, 207-234 (1968)

5. Christodoulou, DM, Graham-Eagle, J, Katatbeh, QD: A program for predicting the intervals of oscillations in the solutions of ordinary second-order linear homogeneous differential equations. Adv. Differ. Equ. (2016). doi:10.1186/s13662-016-0774-x (CGK)

6. Abramowitz, M, Stegun, IA: Handbook of Mathematical Functions with Formulas, Graphs, and Mathematical Tables. Dover, New York (1972)

7. Flügge, S: Practical Quantum Mechanics. Springer, Berlin (1994)

8. Ledoux, V, Van Daele, M, Vanden Berghe, G: CP methods and the evaluation of negative energy Coulomb Whittaker functions. J. Comput. Appl. Math. 183, 168-176 (2005)

9. Noble, CJ: Evaluation of negative energy Coulomb (Whittaker) functions. Comput. Phys. Commun. 159, 55-62 (2004)

10. Hall, RL, Katatbeh, QD: Spectral bounds for the Hellmann potential. Phys. Lett. A 287, 183-186 (2001)

11. Hall, RL, Katatbeh, QD: Spectral bounds for the cutoff Coulomb potential. Phys. Lett. A 294, 163-167 (2002)

12. Ciftci, H, Hall, RL, Katatbeh, QD: Coulomb plus power-law potentials in quantum mechanics. J. Phys. A 36, 7001-7007 (2003) 